\title{
In vitro anti-Toxoplasma gondii efficacy of synthesised benzyltriazole derivatives
}

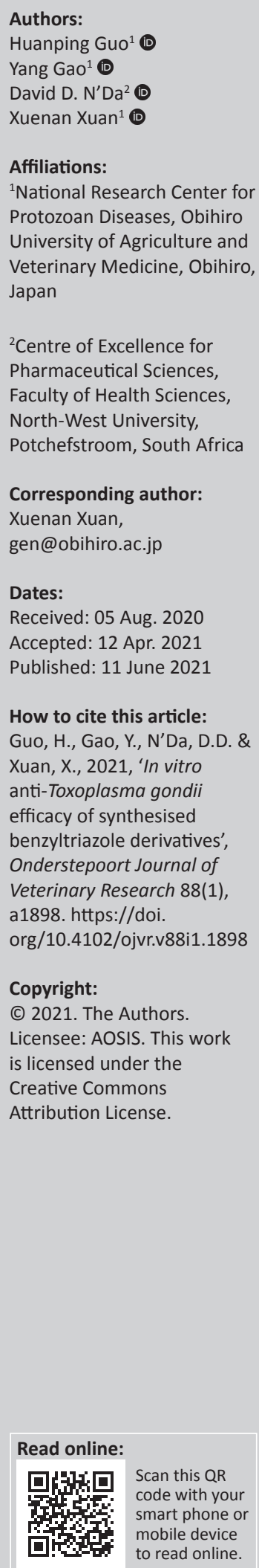

Toxoplasma gondii, an obligate intracellular parasite, is the aetiological agent of toxoplasmosis, a disease that affects approximately $25 \%-30 \%$ of the world's population. At present, no safe and effective vaccine exists for the prevention of toxoplasmosis. Current treatment options for toxoplasmosis are active only against tachyzoites and may also cause bone marrow toxicity. To contribute to the global search for novel agents for the treatment of toxoplasmosis, we herein report the in vitro activities of previously synthesised benzyltriazole derivatives. The effects of these compounds against $T$. gondii in vitro were evaluated by using a expressing green fluorescent protein (GFP) type I strain parasite (RH-GFP) and a type II cyst-forming strain of parasite (Pru $\Delta \mathrm{ku} 80 \Delta \mathrm{hxgprt}$ ). The frontline antitubercular drug isoniazid, designated as Frans J. Smit -isoniazid (FJS-INH), was also included in the screening as a preliminary test in view of future repurposing of this agent. Of the compounds screened, FJS-302, FJS-303, FJS403 and FJS-INH demonstrated $>80 \%$ parasite growth inhibition with $\mathrm{IC}_{50}$ values of $5.6 \mu \mathrm{g} /$ $\mathrm{mL}, 6.8 \mu \mathrm{g} / \mu \mathrm{L}, 7.0 \mu \mathrm{g} / \mathrm{mL}$ and $19.8 \mu \mathrm{g} / \mathrm{mL}$, respectively. FJS-302, FJS-303 and FJS-403 inhibited parasite invasion and replication, whereas, sulphadiazine (SFZ), the positive control, was only effective against parasite replication. In addition, SFZ induced bradyzoite differentiation in vitro, whilst FJS-302, FJS-303 and FJS-403 did not increase the bradyzoite number. These results indicate that FJS-302, FJS-303 and FJS-403 have the potential to act as a viable source of antiparasitic therapeutic agents.

Keywords: toxoplasmosis; tachyzoite; bradyzoite; benzyltriazole; anti-Toxoplasma gondii efficacy; in vitro.

\section{Introduction}

The protozoan parasite Toxoplasma gondii is an obligate intracellular pathogen, which belongs to the phylum Apicomplexa, and virtually infects any kind of warm-blooded animal, including humans (Dubey 2010). Approximately 25\% - 30\% of the world's population is infected with T. gondii (Maenz et al. 2014). The life cycle of T. gondii includes a sexual stage that develops only in definitive hosts such as cat and an asexual stage, which can develop in definitive and intermediate hosts including various warm-blooded animals (Dubey 2010). Asexual reproduction occurs in two phases: firstly, tachyzoites (or endozoites) replicate rapidly by repeated endodyogeny in host cells, and then secondly the next phase starts from the last generation of the tachyzoites that transform into tissue cysts in which bradyzoites (or cystozoites) replicate slowly by endodyogeny (Dubey \& Beattie 1998; Dubey, Lindsay \& Speer 1998). The tachyzoite can cause a strong inflammatory response and tissue destruction and therefore is responsible for clinical manifestations of toxoplasmosis. On the other hand, bradyzoites persist inside cysts for the lifetime of the host (Maenz et al. 2014), and in immunocompromised patients bradyzoites can be released from cysts, transformed back into tachyzoites and can cause reactivation of the infection (Weiss \& Kim 2000).

Primary infection with $T$. gondii in pregnant women or animals can lead to congenital diseases such as hydrocephalus and chorioretinitis in newborn children (Goldstein, Montoya \& Remington 2008). Currently, there is no safe and effective vaccine for preventing toxoplasmosis. An effective chemotherapy constitutes the only alternative to control the disease. Medications expressing antibacterial (sulphadiazine [SFZ], clindamycin and spiramycin) or antimalarial activity (pyrimethamine [PYR] and atovaquone) are the classical chemotherapy (Antczak, Dzitko \& Długońska 2016). It is noteworthy that the therapy of the disease based on these drugs is active only against tachyzoites and limited in eliminating encysted bradyzoites (McLeod et al. 2006). Moreover, the recommended chemotherapy involves a combination of SFZ and PYR. However, synergistic action of SFZ and PYR disturbs folic acid biosynthesis and is also toxic to human cells (Antczak et al. 2016). Therefore, novel efficacious drugs for toxoplasmosis are urgently needed. 
The triazole core, a well-known privileged structure, has drawn much attention in drug discovery, including a fivemember $N$-heterocyclic compound 1,2,3-triazole (Ali et al. 2017; Antczak et al. 2016; Dheer et al. 2017). Many compounds containing 1,2,3-triazole have showed good activities in many different diseases, such as antitubercular (TB) (Boechat et al. 2011), antifungal (Dai et al. 2015), antihuman immunodeficiency virus (HIV) (Mohammed et al. 2016), antimalarial (Kumar et al. 2014a; Singh et al. 2017), antiinflammatory (Shafi et al. 2012) and antitoxoplasmosis activities (Alday \& Doggett 2017; Dzitko et al. 2014; Luan et al. 2019). The moiety possesses hydrogen-bonding capability, moderate dipole character, rigidity and stability under in vivo conditions, which all together are responsible for its enhanced biological properties (Zhang et al. 2017). Interestingly, some compounds containing triazole and isoniazid share a similar mechanism of action as they inhibit microbial cell wall synthesis by blocking lipid biosynthesis (Kumar et al. 2014b; Zhang et al. 2017).

In an effort to contribute to the global search for effective and safe new agents for the treatment of toxoplasmosis, we herein report the in vitro anti-T. gondii efficacy of previously synthesised benzyltriazole (BnTz) derivatives.

\section{Materials and methods Cytotoxicity analysis}

The cytotoxicity of the chemical compounds on human foreskin fibroblasts (HFF) cells was determined by using the cell counting kit-8 (CCK-8) (Dojindo Molecular Technologies, Inc. Japan) according to the manufacturer's instructions. The compounds were dissolved in dimethyl sulfoxide (DMSO). HFF cells were plated in 96-well plates at a density of $1 \times 10^{4}$ cells per well. After a 48 -h incubation at $37^{\circ} \mathrm{C}$ in a $5 \% \mathrm{CO}_{2}$ atmosphere, cells were exposed to various concentrations of tested chemical compounds $(1 \mu \mathrm{g} / \mathrm{mL}, 5 \mu \mathrm{g} / \mathrm{mL}, 10 \mu \mathrm{g} / \mathrm{mL}$, $25 \mu \mathrm{g} / \mathrm{mL}, 50 \mu \mathrm{g} / \mathrm{mL}$ and $100 \mu \mathrm{g} / \mathrm{mL})$. Culture medium containing the same volume of DMSO was used as a negative control. After $24 \mathrm{~h}$, the surviving cells were determined by adding CCK-8 reagent. Cell viability was measured based on the absorbance at $450 \mathrm{~nm}$ after an additional 4-h incubation.

\section{In vitro growth inhibition assay}

To evaluate the activities of synthesised chemical compounds and FJS-INH on T. gondii in vitro, a preliminary screening was performed at a single concentration of $50 \mu \mathrm{g} / \mathrm{mL}$ as previously described (Leesombun et al. 2016). Briefly, HFF cells were seeded into 96-well plates $\left(1 \times 10^{4}\right.$ cells /well $)$ and cultured for $48 \mathrm{~h}$. Expressing green fluorescent protein (GFP) type I strain RH (RH-GFP) tachyzoites (Nishikawa et al. 2003) were added to the wells $\left(5 \times 10^{4}\right.$ tachyzoites/well). After a 4-h incubation, extracellular parasites were removed by washing. Then, chemical compounds at a final concentration of $50 \mu \mathrm{g} / \mathrm{mL}$ were added. Medium and SFZ $(1 \mathrm{mg} / \mathrm{mL})$-treated infected wells were used as negative and positive controls, respectively, whilst uninfected wells treated with 'medium' or 'medium' or 'compounds' were used to correct for background signal. After a 72-h incubation, the fluorescence intensity of RH-GFP was measured to determine the parasite growth. Compounds with parasite inhibition $\geq 80 \%$ were further screened for dose-response effects at final concentrations of $3.125 \mu \mathrm{g} / \mathrm{mL}, 6.25 \mu \mathrm{g} / \mathrm{mL}$, $12.5 \mu \mathrm{g} / \mathrm{mL}, 25 \mu \mathrm{g} / \mathrm{mL}$ and $50 \mu \mathrm{g} / \mathrm{mL}$.

\section{Effects of chemical compounds on Toxoplasma gondii replication in vitro}

To evaluate effect of the screened chemical compounds (FJS-302, FJS-303, FJS-403 and FJS-INH) on parasite replication, Vero cells were plated in 12-well plates at a density of $1 \times 10^{5}$ cells per well and incubated for $24 \mathrm{~h}$ at $37{ }^{\circ} \mathrm{C}$ in a $5 \% \mathrm{CO}_{2}$ atmosphere as previously described. RH-GFP tachyzoites were then added to Vero cells at $1 \mathrm{~mL} /$ well (parasites per host cell ratio $=2: 1$ ). At $2 \mathrm{~h}$ postinfection, the extracellular parasites were removed by washing, and chemical compounds were added (FJS-302, $10 \mu \mathrm{g} / \mathrm{mL}$; FJS-303, $12.5 \mu \mathrm{g} / \mathrm{mL}$; FJS-403, $12.5 \mu \mathrm{g} / \mathrm{mL}$; FJSINH, $50 \mu \mathrm{g} / \mathrm{mL}$ or $1 \mathrm{mg} / \mathrm{mL}$ of SFZ) in Eagle's minimum essential medium (Sigma, United States (US)) (EMEM) (Sigma, United States of America) supplemented with 8\% foetal bovine serum (FBS) (Biowest, Japan). After a 24-h incubation, indirect fluorescent antibody test (IFAT) was performed as previously described (Leesombun et al. 2016) by using anti-surface antigen 1 (SAG1) mouse polyclonal antibody (Guo et al. 2019). Parasite replication in Vero cells was determined by counting the number of tachyzoites per parasitophorous vacuole (PV) (at least 100 vacuoles were randomly selected per well).

\section{Effects of chemical compounds on Toxoplasma gondii invasion in vitro}

Vero cells were seeded on 12-well plates as described here, and purified RH-GFP tachyzoites were treated with FJS-302, FJS-303, FJS-403 or FJS-INH for $1 \mathrm{~h}$ at $37^{\circ} \mathrm{C}$. Then treated parasites were added to Vero cells in a 12-well plate $\left(2 \times 10^{5}\right.$ tachyzoites per well). At $2 \mathrm{~h}$ postinfection, the extracellular parasites were removed by washing, and the infected Vero cells were further incubated for $24 \mathrm{~h}$ at $37^{\circ} \mathrm{C}$ in a $5 \% \mathrm{CO}_{2}$ incubator. After incubation, IFAT was conducted as described here to evaluate the effects of the chemical compounds on parasite invasion. The infection rates were calculated as follows:

$$
\frac{\text { [number of SAG1-positive Vero cells }]}{[100 \text { randomly selected Vero cells }]} \times 100
$$

[Eqn1]

In addition, at least 10 fields were observed per group to measure the average number of parasites per field.

\section{Bradyzoite differentiation assay}

To evaluate the effect of FJS-302, FJS-303, FJS-403 and FJSINH on spontaneously induced bradyzoite differentiation in

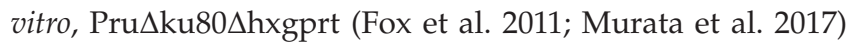
that expresses GFP under the control of bradyzoite-specific gene lactate dehydrogenase 2 (LDH2) promoter was used. 
Vero cells were plated in 12-well plates at a density of $1 \times 10^{5}$ cells per well and incubated for $24 \mathrm{~h}$ at $37{ }^{\circ} \mathrm{C}$ in a $5 \% \mathrm{CO}_{2}$ atmosphere. Then, purified Pru $\Delta$ ku80 $\Delta$ hxgprt tachyzoites were added to Vero cells at $1 \mathrm{~mL} /$ well (parasites per host cell ratio $=1: 1$ ). Two hours postinfection, chemical compounds were added in EMEM supplemented with $8 \%$ FBS. Indirect fluorescent antibody test was conducted after a 48-h incubation. Tachyzoites were stained by using anti-SAG1 mouse polyclonal antibody (Guo et al. 2019), and bradyzoites were detected by using anti-GFP rabbit polyclonal antibody (Medical \& Biological Laboratories CO., LDT, Japan). Secondary antibody Alexa Fluor 594-conjugated goat antimouse IgG (Invitrogen, United States) diluted 1:1000 and Alexa Fluor 488-conjugated goat anti-Rabbit IgG (Invitrogen, United States) diluted 1:1000 were used. Five fields per well were observed by using an All-in-one Fluorescence Microscope (BZ-9000, Keyence, Japan), and the percentage of bradyzoites was calculated.

\section{Statistical analysis}

Statistical analyses were performed by using one-way analysis of variance (ANOVA) followed by the TukeyKramer test for group comparisons. Data were expressed as the mean \pm standard deviation (s.d.). All data were analysed by using GraphPad Prism 8 software (GraphPad Software Inc., United States). A $p$-value of $<0.05$ was considered statistically significant.

\section{Results and discussion \\ Chemistry}

The BnTz derivatives (Table 1) were previously synthesised, and their lipophilicity data have been reported (Smit et al. 2019). However, lipophilicity alongside electronegativity was included in the current investigation as parameters susceptible to impact the antitoxoplasmosis activity of the compounds.

The rationale for introducing the substituents $\mathrm{R}\left(\mathrm{H}, \mathrm{CH}_{3}\right.$, $\mathrm{Br}$ or $\mathrm{CF}_{3}$ ) on the benzene ring was to evaluate the impact the electronic effect might have on the biological activity. The choice of the various electronic groups $\left(\mathrm{Br}\right.$ and $\mathrm{CF}_{3}-$ electron withdrawing groups [EWG] with a destabilising effect, $\mathrm{CH}_{3}$ - electron donating group [EDG] with stabilising effect) was previously justified (Smit et al. 2019). Similarly, the lipophilic side chains were anchored to the triazole ring to assess the influence of lipophilicity of the pharmacological effect of the compounds. Indeed, the $n$-octanol/water partition coefficient Latency/overhead/ gap/Processor $(\log P)$ is a key parameter used for the measurement of the balance between hydrophilicity and lipophilicity. It gives insight into the transport characteristics of a chemical across a biological membrane through passive diffusion (Gombar \& Enslein 1996). Partition coefficient values between 1 and 5 are usually targeted, whilst values between 1 and 3 are ideal (Lipinski et al. 2001). For $n$-alkyl substituted compounds, the derivative lipophilic had positive correlation with the chain length (Table 1).
TABLE 1: Synthesised benzyltriazole derivatives.

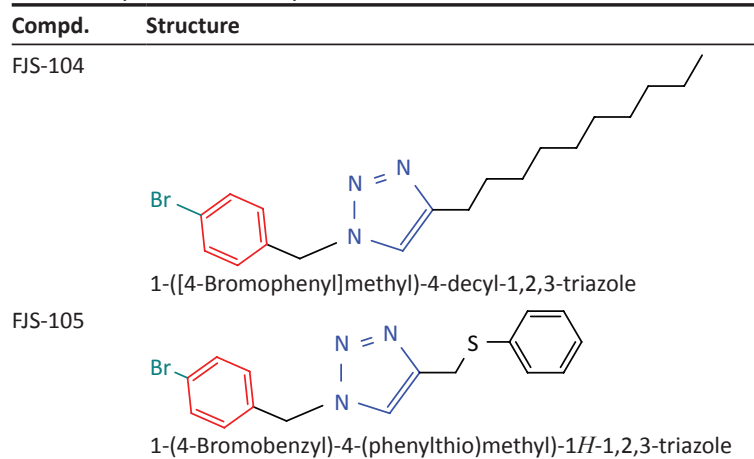

FJS-201

1-(4-Bromobenzyl)-4-(phenylthio)methyl)-1H-1,2,3-triazole

FJS-20

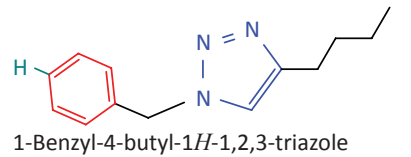

S-205

1-Benzyl-4-butyl-1H-1,2,3-triazole

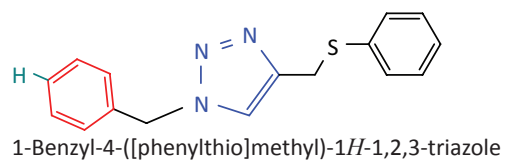

FJS-301

1-Benzyl-4-([phenylthio]methyl)-1H-1,2,3-triazole

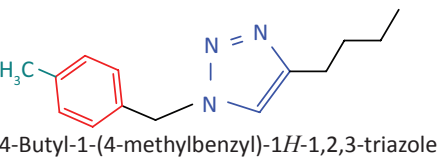

FJS-302

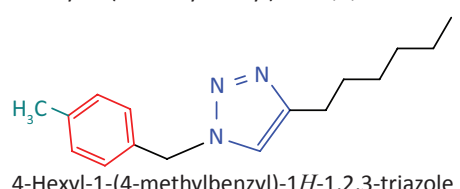

FJS-303

$$
\text { 4-Hexyl-1-(4-methylbenzyl)-1H-1,2,3-triazole }
$$

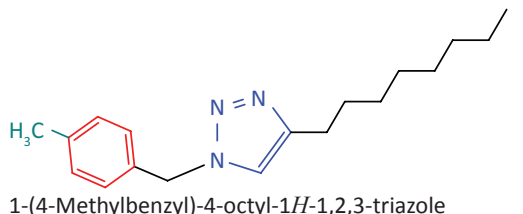

FJS-403

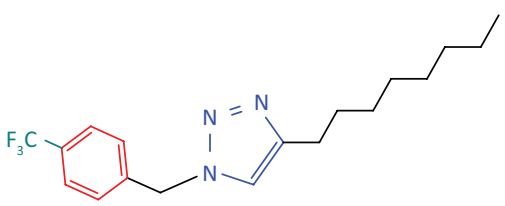

4-Octyl-1-(4-[trifluoromethyl] benzyl)-1H-1,2,3-triazole

FJS-404

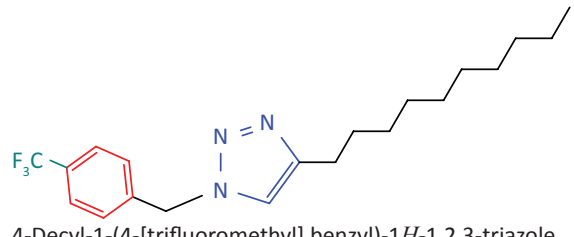

FJS-405

4-Decyl-1-(4-[trifluoromethyl] benzyl)-1H-1,2,3-triazole

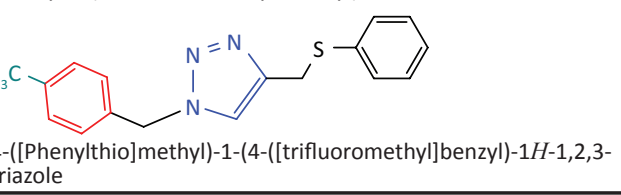

Compd., compound; FJS, Frans J. Smit.

\section{Screening of chemical compounds for anti- Toxoplasma efficacy}

The synthesised BnTz derivatives were screened for their T. gondii growth inhibitory effects at a final concentration of $50 \mu \mathrm{g} / \mathrm{mL}$ alongside the reference drug SFZ (Sanford et al. 2018). The frontline anti-TB drug isoniazid, herein designated 
as FJS-INH (Smit et al. 2019), was also included in the screening as a preliminary test in view of future repurposing of this compound.

TABLE 2: Screening of chemical compounds for anti-RH-GFP efficacy.

\begin{tabular}{|c|c|c|c|c|c|c|}
\hline \multirow{2}{*}{\multicolumn{2}{|c|}{$\begin{array}{l}\text { Compound } \\
\text { Entry Code }\end{array}$}} & \multirow[t]{2}{*}{ cLogP $\uparrow$} & \multicolumn{2}{|c|}{ Anti-toxoplasmosis activity } & \multirow{3}{*}{$\begin{array}{l}\begin{array}{l}\text { \% viability } \\
\text { HFF§ }\end{array} \\
100.03\end{array}$} & \multirow{3}{*}{$\begin{array}{c}\begin{array}{c}\% \\
\text { viability } \\
\text { Veroף }\end{array} \\
102.65\end{array}$} \\
\hline & & & \multirow{2}{*}{$\begin{array}{c}\begin{array}{c}\% \text { Parasite inhibition } \\
\text { (mean } \pm \text { s.d.) } \$\end{array} \\
62.2 \pm 6.0\end{array}$} & \multirow{2}{*}{$\frac{\mathrm{IC}_{50}(\mu \mathrm{g} / \mathrm{mL})}{\mathrm{ND}}$} & & \\
\hline 1 & FJS-104 & 7.13 & & & & \\
\hline 2 & FJS-105 & 4.23 & $60.5 \pm 6.1$ & ND & 100.04 & 103.31 \\
\hline 3 & FJS-201 & 1.95 & $64.6 \pm 6.8$ & ND & 100.02 & 99.34 \\
\hline 4 & FJS-205 & 2.22 & $60.2 \pm 5.1$ & ND & 100.08 & 100.66 \\
\hline 5 & FJS-301 & 3.59 & $57.7 \pm 3.0$ & ND & 96.16 & 85.43 \\
\hline 6 & FJS-302 & 4.65 & $95.9 \pm 4.6$ & 5.6 & 85.00 & 86.09 \\
\hline 7 & FJS-303 & 5.71 & $96.3 \pm 3.9$ & 6.8 & 110.35 & 100.66 \\
\hline 8 & FJS-403 & 6.09 & $91.5 \pm 1.7$ & 7.0 & 103.47 & 101.97 \\
\hline 9 & FJS-404 & 7.15 & $64.5 \pm 1.8$ & ND & 100.05 & ND \\
\hline 10 & FJS-405 & 4.25 & $35.0 \pm 5.0$ & ND & 100.23 & 103.29 \\
\hline 11 & FJS-INH & -0.67 & $87.2 \pm 4.6$ & 19.8 & 118.26 & 98.03 \\
\hline 12 & SFZ & 0.1 & $73.7 \pm 4.6$ & 59.5 & 105.19 & 92.11 \\
\hline
\end{tabular}

RH-GFP, expressing green fluorescent protein (GFP) type I strain RH; FJS, Frans J. Smit; FJSINH, Frans J. Smit -isoniazid; SFZ, sulphadiazine; HFF, human foreskin fibroblasts; cLogP, INH, Frans J. Smit -isoniazid; SFZ, sulphadiazine; HFF, human foreskin fibroblasts; CLogP,
Latency/overhead/gap/Processor; IC F $_{50}$, half-maximal inhibitory concentration; ND, not Latency/overhead/gap/Processor; ${ }^{I} \mathrm{C}_{50}$
determined; s.d., standard deviation.

$\dagger$, Calculated from ChemDraw. Ultra 12; $\ddagger$, Parasite growth inhibition values of parasiteinfected cells with test chemical compounds at a concentration of $50 \mu \mathrm{g} / \mathrm{mL}$ and sulphadiazine at a concentration of $1 \mathrm{mg} / \mathrm{mL} ; \S$, HFF cell viability values with test chemical compounds at a concentration of $50 \mu \mathrm{g} / \mathrm{mL}$ and sulphadiazine at a concentration of $1 \mathrm{mg} /$ $\mathrm{mL}$; I, Vero Cell viability values with test chemical compounds at a concentration of $100 \mu \mathrm{g} /$ $\mathrm{mL}$ and sulphadiazine at a concentration of $1 \mathrm{mg} / \mathrm{mL}$.
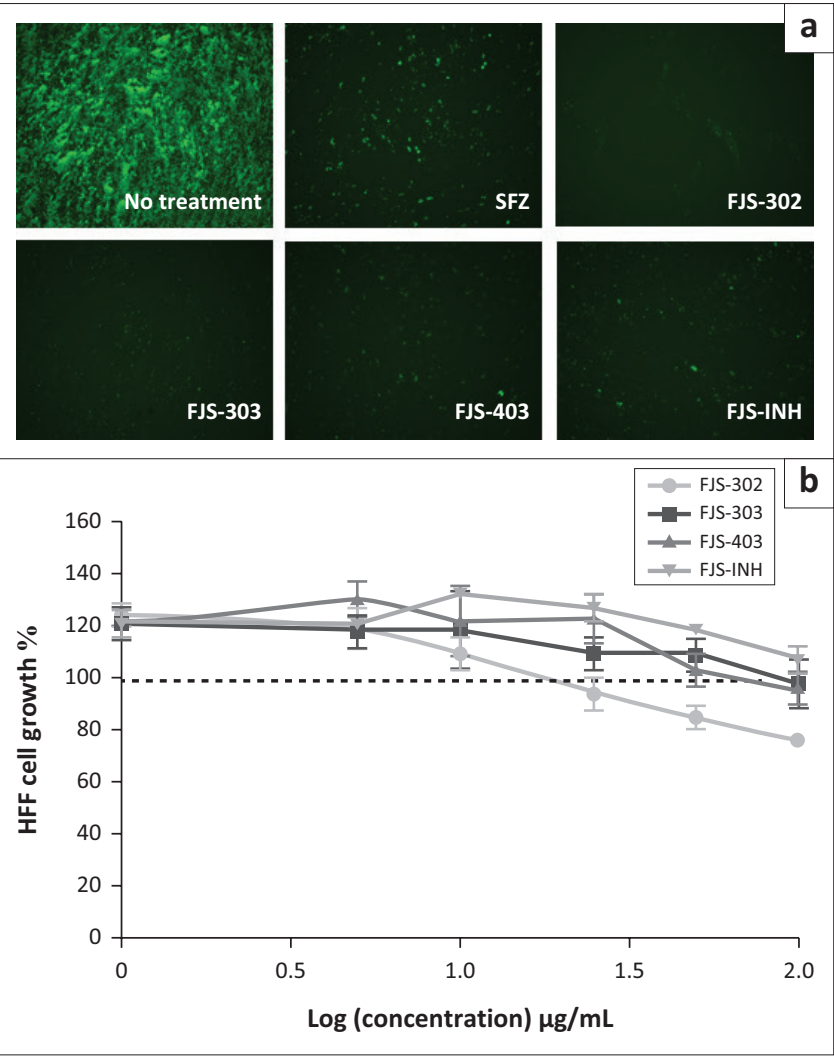

Source: Guo, H., Gao, Y., Jia, H., Moumouni, P.F.A., Masatani, T., Liu, M. et al., 2019, 'Characterization of strain-specific phenotypes associated with knockout of dense granule protein 9 in Toxoplasma gondii', Molecular and Biochemical Parasitology 229, 53-61. https:// doi.org/10.1016/j.molbiopara.2019.01.003

s.d., standard deviation; HFF, human foreskin fibroblasts; SFZ, sulphadiazine; FJS-INH, Frans J. Smit -isoniazid.

FIGURE 1: Anti-Toxoplasma gondii activity of FJS-302, FJS-303, FJS-403 and FJS INH on RH-GFP and their cytotoxicity. (a) Representative images of T. gondii RHGFP-infected HFF cells treated for $72 \mathrm{~h}$ with sulphadiazine $(1 \mathrm{mg} / \mathrm{mL})$, FJS-302 $(50 \mu \mathrm{g} / \mathrm{mL})$, FJS-303 $(50 \mu \mathrm{g} / \mathrm{mL})$, FJS-403 $(50 \mu \mathrm{g} / \mathrm{mL})$ or FJS-INH $(50 \mu \mathrm{g} / \mathrm{mL})$ (b) Cytotoxicity testing of HFF cells after treatment with FJS-302, FJS-303, FJS-403 or 作 $0 \mu \mathrm{g} / \mathrm{mL}$ to $100 \mu \mathrm{g} / \mathrm{mL}$ for $24 \mathrm{~h}$. Data represent the mean values \pm s.d. of three independent experiments.
The average inhibition rate of $\mathrm{BnTz}$ derivatives was $60 \%$ on parasites, whilst FJS-303 showed the highest inhibition with 96\%. Three derivatives, namely FJS-302, FJS-303, FJS-403 and the isoniazid FJS-INH, had a parasite growth inhibition rate $>85 \%$ (Table 2). Parasite growth at $72 \mathrm{~h}$ after infection (Figure 1a) indicates that these three chemical compounds showed better growth inhibitory efficacy than positive control SFZ (1 mg/mL).

The effect of FJS-302, FJS-303, FJS-403 and FJS-INH on host cell viability was examined, and no significant suppression on host cell growth was observed, even at $100 \mu \mathrm{g} / \mathrm{mL}$ (Figure 1b), suggesting that the effect of these BnTz compounds on RHGFP growth was not a consequence of host cell cytotoxicity. Consequently, the parasite inhibitory effects of the screened compounds at different concentrations were evaluated. The $\mathrm{IC}_{50}$ values of FJS-302, FJS-303 and FJS-403 were $5.6 \mu \mathrm{g} / \mathrm{mL}, 6.8$ $\mu \mathrm{g} / \mathrm{mL}$ and $7.0 \mu \mathrm{g} / \mathrm{mL}$, respectively (Figure 2), whilst the $\mathrm{IC}_{50}$ value of SFZ was $59.5 \mu \mathrm{g} / \mathrm{mL}$ (Table 2). The inhibition ratios of FJS-104 to FJS-301, FJS-404 and FJS-405 were lower than that of SFZ, so their $\mathrm{IC}_{50}$ value was not evaluated.

De Oliveira et al. (2009) reported that the $\mathrm{IC}_{50}$ value of SFZ on $\mathrm{RH}$ strain was $70 \mu \mathrm{g} / \mathrm{mL}$, and the viability of HFF cells decreased by $28 \%$ in the presence of $200 \mu \mathrm{g} / \mathrm{mL}$ of SFZ. Thus, FJS-302, FJS-303, FJS-403 and FJS-INH should be more effective at controlling the growth of T. gondii in vitro than SFZ.

The electronic effect had limited impact on the activity of the derivatives. Indeed, in comparing the $\mathrm{IC}_{50}$ values of the active compounds, it can be seen (Table 1 ) that there are no significant differences amongst FJS-302 ( $\left.\mathrm{IC}_{50} 5.6 \mu \mathrm{g} / \mathrm{mL}\right)$, FJS-303 $\left(\mathrm{IC}_{50} 6.8\right.$ $\mu \mathrm{g} / \mathrm{mL}$ ) and FJS-403 ( $\left.\mathrm{IC}_{50} 7.0 \mu \mathrm{g} / \mathrm{mL}\right)$. Similarly, the lipophilicity had marginal influence on compound activity. Indeed, based on the cLogP and $\mathrm{IC}_{50}$ values, the three active $\mathrm{BnTz}$ derivatives had comparable lipophilicity and activities against $T$. gondii. However, a realistic conclusion on structureactivity relationship of the compounds can only be drawn through investigation of a wider series of $\mathrm{BnTz}$ derivatives.

Another interesting finding of this study is the activity of isoniazid. In vitro inhibition of this mainstay anti-TB drug against $T$. gondii has previously been reported (Sanford et al. 2018). In this study, FJS-INH was efficient on T. gondii growth inhibition, although threefold less potent than the leading BnTz derivative. The activity of FJS-INH $\left(\mathrm{IC}_{50}\right.$ of $19.8 \mu \mathrm{g} / \mathrm{mL}$ ) may be suggestive of the potential of alternative use as antitoxoplasmosis agent. Thus, further optimisation through investigation of novel derivatives of FJS-INH for treatment of toxoplasmosis may be worth it.

\section{Effects of chemical compounds on parasite invasion and in vitro replication}

To test whether these compounds are active on inhibition of parasite invasion, purified parasites were pretreated with FJS-302 (10 $\mu \mathrm{g} / \mathrm{mL})$, FJS-303 (12.5 $\mu \mathrm{g} / \mathrm{mL})$, FJS-403 $(12.5 \mu \mathrm{g} / \mathrm{mL})$, FJS-INH $(50 \mu \mathrm{g} / \mathrm{mL})$ or $1 \mathrm{mg} / \mathrm{mL}$ of $\mathrm{SFZ}$ before the infection of Vero cells. Infection rate of non-treated 


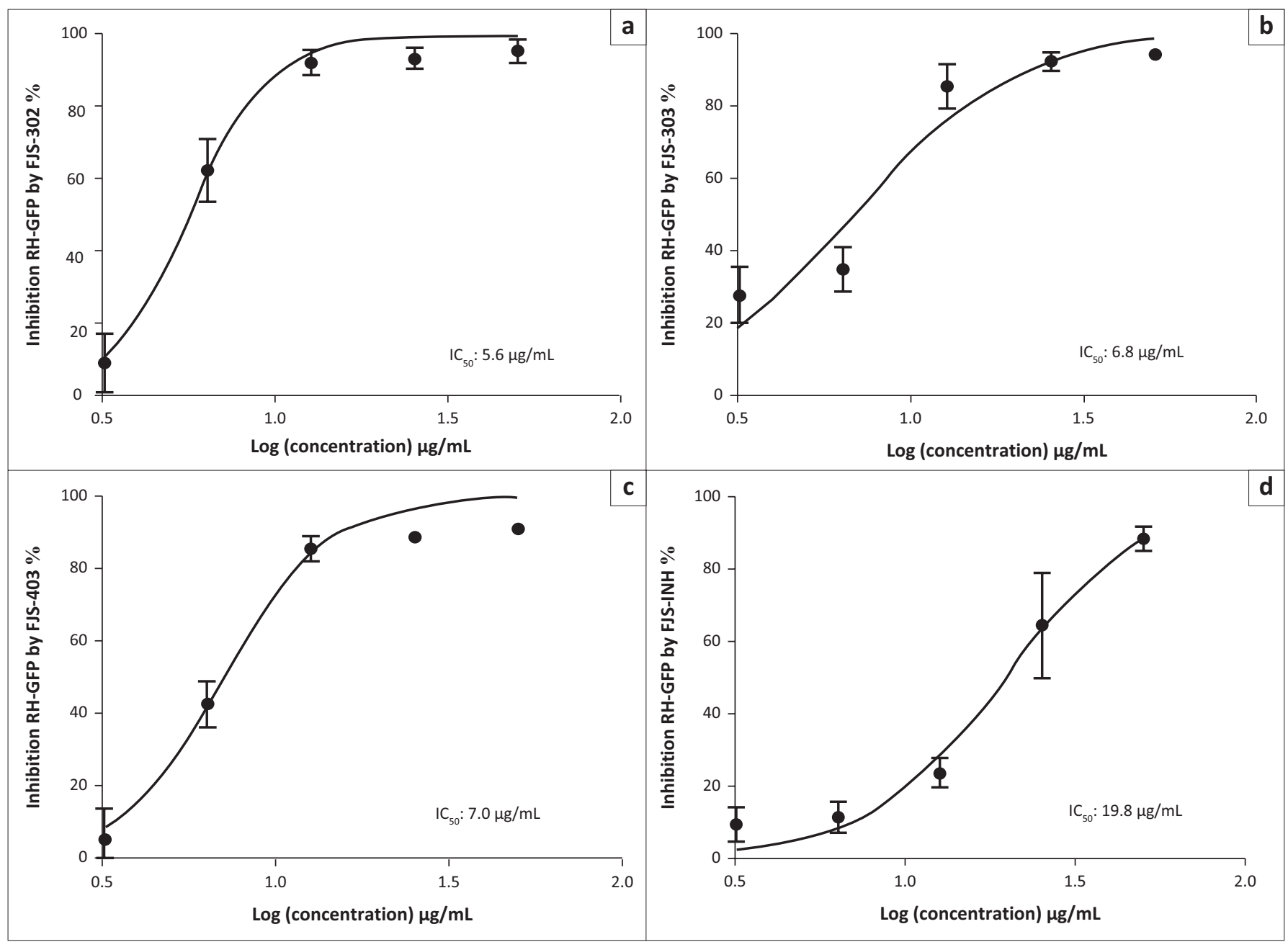

GFP, green fluorescent protein; FJS, Frans J. Smit; IC s0 , half-maximal inhibitory concentration; HFF, human foreskin fibroblasts.

FIGURE 2: The IC $\mathrm{C}_{50}$ values of (a) FJS-302, (b) FJS-303, (c) FJS-403 and (d) FJS-INH on Toxoplasma gondii RH-GFP. The RH-GFP-infected HFF cells were treated with the given chemical compounds for $72 \mathrm{~h}$ at different concentrations from $3.125 \mu \mathrm{g} / \mathrm{mL}$ to $50 \mu \mathrm{g} / \mathrm{mL}$. Data represent the mean values \pm s.d. of three independent experiments.

cell was $11.3 \% \pm 0.6 \%$. Pretreatment with FJS-302, FJS-303 and FJS-403 significantly decreased the infection rates to $6.2 \% \pm 0.5 \%, 5.8 \% \pm 0.5 \%$ and $5.2 \% \pm 0.7 \%$, respectively, whilst pretreatment with FJS-INH and SFZ showed $10.7 \% \pm 0.5 \%$ and $11.7 \% \pm 1.3 \%$ infection rates (Figure 3a). At the same time, the number of parasites per field after pretreatment with FJS-302, FJS-303 or FJS-403 was also significantly reduced (Figure $3 b$ ), suggesting that these $\mathrm{BnTz}$ derivatives were effective in inhibiting the invasion of extracellular parasites.

To examine the effect of the four chemical compounds on parasite replication, RH-GFP-infected Vero cells were treated with the same concentration of chemical compounds or SFZ. The number of tachyzoites per PV was counted (Figure 3c). The percentage of PV containing four or more tachyzoites was $78.7 \%$ in no treatment group whilst this percentage was lower in the groups treated with chemical compounds or SFZ (Figure 3d). In addition, the average number of tachyzoites per PV after treatment was significantly reduced compared with the no treatment group (Figure 3e). These data indicate that all four chemical compounds inhibited parasite replication.
The different life stages of T. gondii are vital for the parasite survival in intermediate and definitive hosts; however, the tachyzoite is the rapidly multiplying stage of the parasite (Maenz et al. 2014). Through their rapid replication, tachyzoites can damage host tissue. FJS-302, FJS-303 and FJS-403 were effective against extra- and intracellular parasites, whilst SFZ affected only intracellular parasite.

\section{Effects of chemical compounds on bradyzoite induction}

To determine whether the BnTz derivatives-treated tachyzoite could induce bradyzoite differentiation, we assessed the bradyzoites ratio of Pru $\Delta$ ku80 $\Delta$ hxgprt, a T. gondii strain that can undergo spontaneous bradyzoite differentiation (Murata et al. 2017) when parasites are incubated at their most effective concentration. Indirect fluorescent antibody test was performed to determine the number of tachyzoites and bradyzoites after $48 \mathrm{~h}$ of treatment of the infected cells with specific concentration of the four chemical compounds and SFZ (Figure 4a and 4b). Parasite growth was estimated by the total number of tachyzoite and bradyzoite in each field (Figure 4b). All tested compounds 


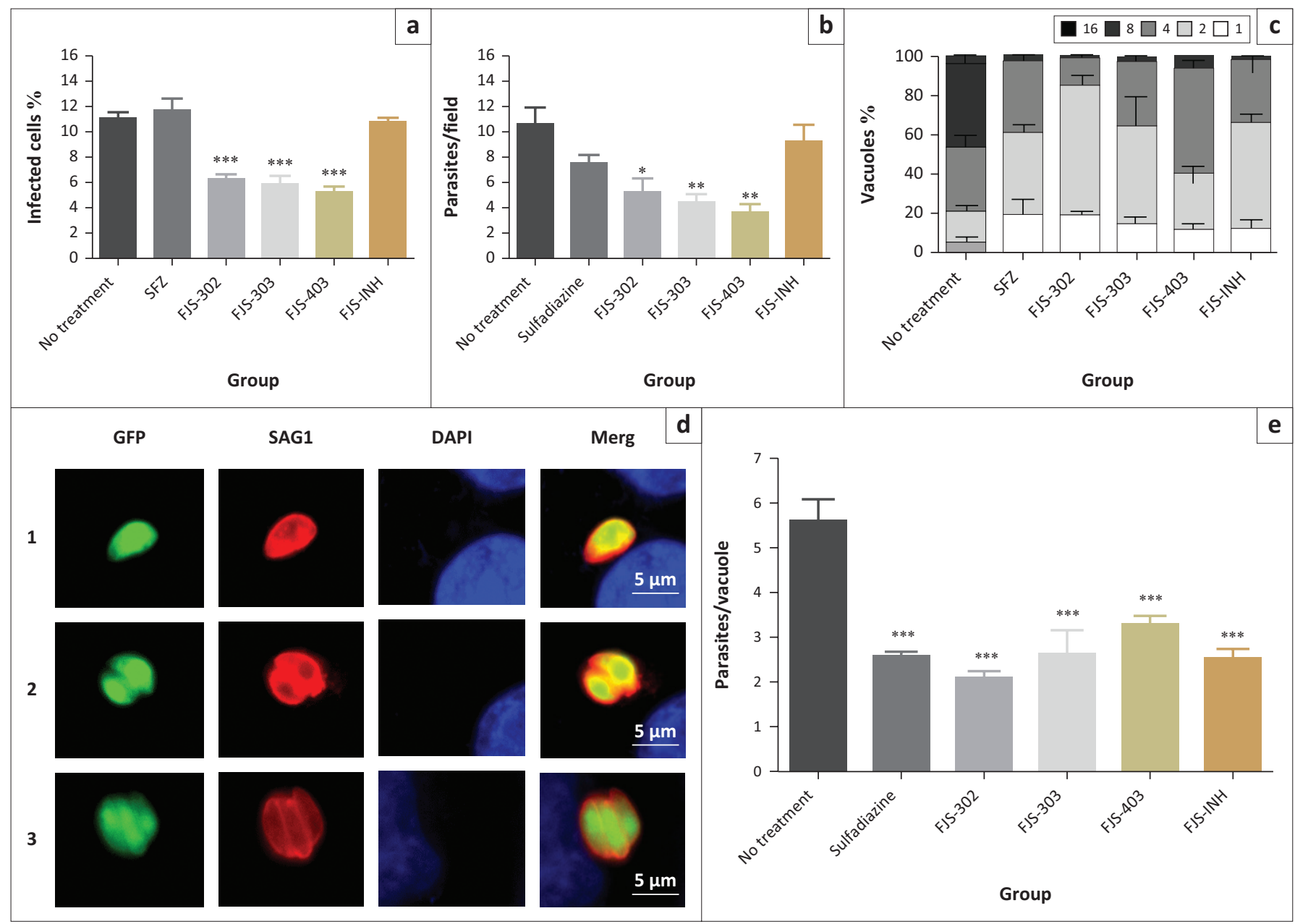

FJS, Frans J. Smit; FJS-INH, Frans J. Smit -isoniazid; SFZ, sulphadiazine; SAG1, surface antigen 1; RH-GFP, expressing green fluorescent protein (GFP) type I strain RH; PV, parasitophorous vacuole; s.d., standard deviation.

$*, p<0.05 ; * *, p<0.01 ; * * *, p<0.0001$.

FIGURE 3: Effects of FJS-302, FJS-303, FJS-403 and FJS-INH on Toxoplasma gondii invasion and replication. FJS-302 (10 mg/mL), FJS-303 (12.5 mg/mL), FJS-403 (12.5 mg/ $\mathrm{mL})$, FJS-INH $(50 \mathrm{mg} / \mathrm{mL}$ ) and sulphadiazine $(1 \mathrm{mg} / \mathrm{mL})$. (a) The percentage of infected cells was evaluated by counting the number of SAG1-positive Vero cells per 100 Vero cells by using IFAT. Each bar represents the mean \pm s.d. of three independent experiments. (b) The number of parasites per field was measured by using a microscope. At least 10 fields were randomly observed in each group. Each bar represents the mean \pm s.d. of three independent experiments. (c) Representative images of $T$. gondii RH-GFP replication in Vero cells. (d) The number of parasites in parasitophorous vacuoles was measured by counting the number of SAG1-positive parasites per parasitophorous vacuole. Each bar represents the mean \pm s.d. of three independent experiments. (e) The average number of parasites per parasitophorous vacuole. Each bar represents the mean \pm s.d. of three independent experiments.

reduced the total parasite and tachyzoite numbers. However, bradyzoite number in the SFZ group $(23.7 \pm 6.0)$ was higher than that of the no treatment group $(6.5 \pm 1.5)$. Regarding the bradyzoite rate, SFZ significantly induced bradyzoite differentiation $(46.6 \% \pm 7.9 \%)$, whereas none of the four chemical compounds induced a bradyzoite differentiation. Bradyzoite differentiation in compound-treated group was comparable to the no treatment group $(8.2 \% \pm 1.9 \%)$ (Figure 4c). This demonstrates that the four chemical compounds are not selective for tachyzoites and have no bradyzoite-inducing effect.

Whilst some therapy exists for the treatment of acute $T$. gondii infection, it is necessary to develop new therapeutic agents that are active against both acute and chronic infection and have mild side effects and low toxicity on host cells. Pru $\Delta$ ku80 $\Delta$ hxgprt with a bradyzoite reporter is useful for the evaluation of chemical compounds for their effects on both tachyzoites and bradyzoites (Murata et al.
2017). Here, FJS-302, FJS-303, FJS-403 and FJS-INH had significant antitachyzoite activity and a degree of noninducing effect on bradyzoites. Agents that can eliminate tachyzoites and bradyzoites may be used to treat both acute and chronic infection. Several compounds that target the replication of bradyzoites have been identified (Doggett et al. 2014; Murata et al. 2017). The effects of these chemical compounds against $T$. gondii in vivo should also be evaluated.

\section{Conclusion}

In this study, a series of previously synthesised BnTz derivatives, FJS-302, FJS-303, FJS-403 and FJS-INH inhibited tachyzoite growth. FJS-302, FJS-303 and FJS-403 were effective against extra- and intracellular parasites, whilst the current recommended drug SFZ affected only intracellular parasite. In addition, SFZ induced bradyzoite differentiation, whereas FJS-302, FJS-303 and FJS-403 did not increase the bradyzoite number. These results 

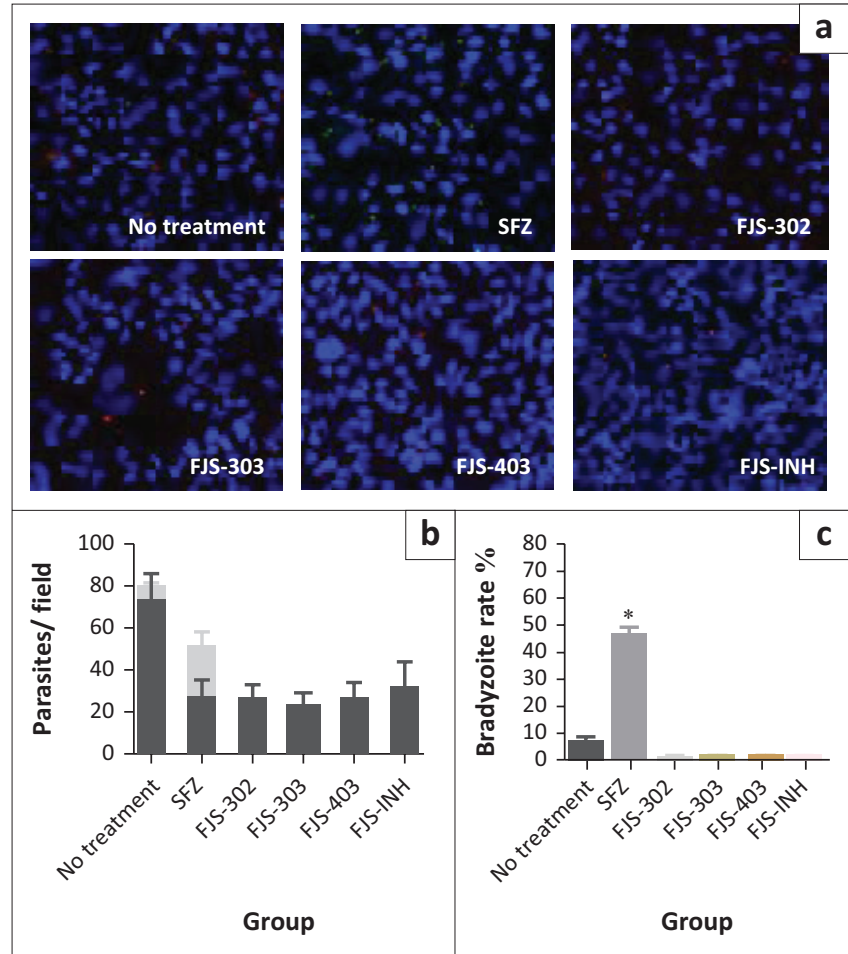

FJS, Frans J. Smit; FJS-INH, Frans J. Smit -isoniazid; SFZ, sulphadiazine; SAG1, surface antigen 1; DAPI, 4',6-diamidino-2-phenylindole; RH-GFP, expressing green fluorescent protein (GFP) type I strain RH; PV, parasitophorous vacuole; s.d., standard deviation.

$*, p<0.0001$.

FIGURE 4: Effects of FJS-302, FJS-303, FJS-403 and FJS-INH on Toxoplasma gondii bradyzoite induction. (a) Representative images of the sulphadiazine $(1 \mathrm{mg} / \mathrm{mL})$, FJS-302 $(50 \mu \mathrm{g} / \mathrm{mL})$, FJS-303 $(50 \mu \mathrm{g} / \mathrm{mL})$, FJS-403 $(50 \mu \mathrm{g} / \mathrm{mL})$ or FJS-INH $(50 \mu \mathrm{g} / \mathrm{mL})$ treated wells. Red, SAG1; Green, GFP; Blue, DAPI. (b) GFP-positive parasitophorous vacuole (bradyzoite) number and anti-SAG1 positive parasitophorous vacuole (tachyzoite) number were counted. (c) GFP-positive parasitophorous vacuole rate was calculated by counting total parasitophorous vacuole and GFP-positive parasitophorous vacuole per image. Each bar represents the mean \pm s.d. of three independent experiments.

indicate that FJS-302, FJS-303 and FJS-403 have the potential to act as a viable source of antiparasitic therapeutic agents.

\section{Acknowledgements}

We thank Dr. Arpron Leesombun and Dr. Yong mei Han from the National Research Center for Protozoan Diseases, Obihiro University of Agriculture and Veterinary Medicine for support in conducting cytotoxicity analysis and bradyzoite differentiation assay experiments; thank you to Frans J. Smit from North-West University for the support on the compounds synthesised work.

\section{Competing interests}

The authors declare that they have no financial or personal relationships that may have inappropriately influenced them in writing this article.

\section{Authors' contributions}

D.D.N. and X.X. were responsible for the conceptualisation and design of the study. H.G. and Y.G. performed the antitoxoplasmosis experiments, analysed and interpreted the data. D.D.N., H.G. and Y.G. drafted the manuscript, developed the figures and tables. All authors were involved in revising and approved the final version of the manuscript.

\section{Ethical considerations}

This article followed all ethical standards for a research without direct contact with human or animal subjects.

\section{Funding information}

This study was supported by a Grant-in-Aid for Scientific Research, from the Ministry of Education, Culture, Sports, Science, and Technology of Japan (to XX;18H02336). This collaboration work was funded by grants from the South African National Research Foundation (NRF) (to DD N'Da; UID 76443; 98937).

\section{Data availability}

Data sharing is not applicable to this article as no new data were created or analysed in this study.

\section{Disclaimer}

The views and opinions expressed in this article are those of the authors and do not necessarily reflect the official policy or position of any affiliated agency of the authors.

\section{References}

Alday, P.H. \& Doggett, J.S., 2017, 'Drugs in development for toxoplasmosis: Advances, challenges, and current status', Drug Design, Development and Therapy 11, 273-293. https://doi.org/10.2147/DDDT.S60973

Ali, A.A., Gogoi, D., Chaliha, A.K., Buragohain, A.K., Trivedi, P., Saikia, P.J. et al., 2017, 'Synthesis and biological evaluation of novel 1,2,3-triazole derivatives as antitubercular agents', Bioorganic \& Medicinal Chemistry Letters 27(16), 3698-3703. https://doi.org/10.1016/j.bmcl.2017.07.008

Antczak, M., Dzitko, K. \& Długońska, H., 2016, 'Human toxoplasmosis-searching for novel chemotherapeutics', Biomedicine \& Pharmacotherapy 82, 677-684. https:// doi.org/10.1016/j.biopha.2016.05.041

Boechat, N., Ferreira, V.F., Ferreira, S.B., Ferreira, M.D.L.G., Da Silva, F.D.C., Bastos, M.M. et al., 2011, 'Novel 1,2,3-triazole derivatives for use against Mycobacterium tuberculosis H37Rv (ATCC 27294) strain', Journal of Medicinal Chemistry 54(17), tuberculosis H37Rv (ATCC 27294) strain', Journal
5988-5999. https://doi.org/10.1021/jm2003624

Dai, Z.C., Chen, Y.F., Zhang, M., Li, S.K., Yang, T.T., Shen, L. et al., 2015, 'Synthesis and antifungal activity of 1, 2, 3-triazole phenylhydrazone derivatives', Organic \& Biomolecular Chemistry 13(2), 477-486. https://doi.org/10.1039/ \& Bob01758g

De Oliveira, T.C., Silva, D.A., Rostkowska, C., Béla, S.R., Ferro, E.A., Magalhães, P.M et al., 2009, 'Toxoplasma gondii: Effecrs of Artemisia annua L. on susceptibility to infection in experimental models in vitro and vivo', Experimental Parasitology 122 (3), 233-241. https://doi.org/10.1016/j.exppara.2009.04.010

Dheer, D., Singh, V. \& Shankar, R., 2017, 'Medicinal attributes of 1,2,3-triazoles: Current developments', Bioorganic Chemistry 71, 30-54. https://doi. org/10.1016/j.bioorg.2017.01.010

Doggett, J.S., Ojo, K.K., Fan, E., Maly, D.J. \& Van Voorhis, W.C., 2014, 'Bumped kinase inhibitor 1294 treats established Toxoplasma gondii infection', Antimicrobial Agents and Chemotherapy 58(6), 3547-3549. https://doi.org/10.1128/AAC.01823-13

Dubey, J.P., 2010, 'Toxoplasmosis of animals and humans, second edition', Journal of Parasitology 96(5), 940. https://doi.org/10.1645/GE-2605.1

Dubey, J.P. \& Beattie, C.P., 1998, Toxoplasmosis of animals and humans, CRC Press, Boca Raton, FL.

Dubey, J.P., Lindsay, D.S. \& Speer, C.A., 1998, 'Structures of Toxoplasma gondi tachyzoites, bradyzoites, and sporozoites and biology and development of tissue cysts', Clinical Microbiology Reviews 11(2), 267-299. https://doi.org/10.1128/ CMR.11.2.267

Dzitko, K., Paneth, A., Plech, T. Pawełczyk, J., Weglińska, L. \& Paneth, P., 2014 'Triazole-based compound as a candidate to develop novel medicines to treat Toxoplasmosis', Antimicrobial Agents and Chemotherapy 58(12), 7583. https:// doi.org/10.1128/AAC.03832-14 
Fox, B.A., Falla, A., Rommereim, L.M., Tomita, T., Gigley, J.P., Mercier, C. et al., 2011 'Type II Toxoplasma gondii KU80 knockout strains enable functional analysis of genes required for cyst development and latent infection', Eukaryotic Cell 10(9), 1193-1206. https://doi.org/10.1128/EC.00297-10

Goldstein, E.J.C., Montoya, J.G. \& Remington, J.S., 2008, 'Management of Toxoplasma gondii infection during pregnancy', Clinical Infectious Diseases 47(4), 554-566. https://doi.org/10.1086/590149

Gombar, V.K. \& Enslein, V.K., 1996, 'Assessment of n-octanol/water partition coefficient: When is the assessment reliable', Journal of Chemical Information and Computer Sciences 36(6), 1127-1134. https://doi.org/10.1021/ci960028n

Guo, H., Gao, Y., Jia, H., Moumouni, P.F.A., Masatani, T., Liu, M. et al., 2019 "Characterization of strain-specific phenotypes associated with knockout of dense granule protein 9 in Toxoplasma gondii', Molecular and Biochemical Parasitology 229, 53-61. https://doi.org/10.1016/j.molbiopara.2019.01.003

Kumar, D., Beena, Khare, G., Kidwai, S., Tyagi, A.K., Singh, R. et al., 2014a, 'Synthesis of novel 1,2,3-triazole derivatives of isoniazid and their in vitro and in vivo antimycobacterial activity evaluation', European Journal of Medicinal Chemistry 81, 301-313. https://doi.org/10.1016/j.ejmech.2014.05.005

Kumar, K., Pradines, B., Madamet, M., Amalvict, R., Benoit, N. \& Kumar, V., 2014b, ' $1 \mathrm{H}-1,2,3$-triazole tethered isatin-ferrocene conjugates: Synthesis and in vitro antimalarial evaluation', European Journal of Medicinal Chemistry 87, 801-804. https://doi.org/10.1016/j.ejmech.2014.10.024

Leesombun, A., Boonmasawai, S., Shimoda, N. \& Nishikawa, Y., 2016, 'Effects of extracts from Thai piperaceae plants against infection with Toxoplasma gondi', PLoS One 11(5), e0156116. https://doi.org/10.1371/journal.pone.0156116

Lipinski, C.A., Lombardo, F., Dominy, B.W. \& Feeney, P.J., 2001, 'Experimental and computational approaches to estimate solubility and permeability in drug discovery and development settings', Advanced Drug Delivery Reviews 46(1-3), 3-25. https://doi.org/10.1016/s0169-409x(00)00129-0

Luan, T., Jin, C., Jin, C.M., Gong, G.H. \& Quan, Z.S., 2019, 'Synthesis and biological evaluation of ursolic acid derivatives bearing triazole moieties as potential antiToxoplasma gondii agents', Journal Enzyme Inhibition and Medicinal Chemistry 34(1), 761-772. https://doi.org/10.1080/14756366.2019.1584622

Maenz, M., Schlüter, D., Liesenfeld, O., Schares, G., Gross, U. \& Pleyer, U., 2014, 'Ocular toxoplasmosis past, present and new aspects of an old disease', Progress in Retinal and Eye Research 39, 77-106. https://doi.org/10.1016/j.preteyeres.2013.12.005
McLeod, R., Boyer, K., Karrison, T., Kasza, K., Swisher, C., Roizen, N. et al., 2006, 'Outcome of treatment for congenital toxoplasmosis, 1981-2004: The national collaborative Chicago-based, congenital toxoplasmosis study', Clinical Infectious Diseases 42(10), 1383-1394. https://doi.org/10.1086/501360

Mohammed, I., Kummetha, I.R., Singh, G., Sharova, N., Lichinchi, G., Dang, J. et al., 2016, ' $1,2,3$-Triazoles as amide bioisosteres: Discovery of a new class of potent HIV-1 Vif antagonists', Journal of Medicinal Chemistry 59(16), 7677-7682. https:// doi.org/10.1021/acs.jmedchem.6b00247

Murata, Y., Sugi, T., Weiss, L.M. \& Kato, K., 2017, 'Identification of compounds that suppress Toxoplasma gondii and bradyzoites', PLoS One 12(6), e0178203. https:// doi.org/10.1371/journal.pone.0178203

Nishikawa, Y., Xuenan, X., Makala, L., Vielemeyer, O., Joiner, K.A. \& Nagasawa, H., 2003, 'Characterisation of Toxoplasma gondii engineered to express mouse interferon-gamma', International Journal for Parasitology 33(13), 1525-1535. https://doi.org/10.1016/s0020-7519(03)00204-2

Sanford, A.G., Schulze, T.T., Potluri, L.P., Watson, G.F., Darner, E.B., Zach, S.J. et al., 2018, 'Derivatives of a benzoquinone acyl hydrazone with activity against Toxoplasma gondii', Inter, Resistance 8(3), 488-492. https://doi.org/10.1016/j.ijpddr.2018.11.001

Shafi, S., Alam, M.M., Mulakayala, N., Mulakayala, C., Vanaja, G., Kalle, A.M. et al., 2012, 'Synthesis of novel 2-mercapto benzothiazole and 1,2,3-triazole based bisheterocycles: Their anti-inflammatory and anti-nociceptive activities', European Journal of Medicinal Chemistry 49, 324-333. https://doi.org/10.1016/j. eimech.2012.01.032

Singh, A., Gut, J., Rosenthal, P.J. \& Kumar, V., 2017, '4-Aminoquinoline-ferrocenylchalcone conjugates: Synthesis and anti-plasmodial evaluation', European Journal of Medicinal Chemistry 125, 269-277. https://doi.org/10.1016/j.ejmech. 2016.09.044

Smit, F.J., Seldon, R., Aucamp, J., Jordaan, A., Warner, D.F. \& N'Da, D.D., 2019, 'Synthesis and antimycobacterial activity of disubstituted benzyltriazoles', Medicinal Chemistry Research 28, 2279-2293. https://doi.org/10.1007/s00044-019-02458-7

Weiss, L.M. \& Kim, K., 2000, 'The development and biology of bradyzoites of Toxoplasma gondii', Frontiers in Bioscience 5(3), D391-D405. https://doi. org/10.2741/Weiss

Zhang, S., Xu, Z., Gao, C., Ren, Q.C., Chang, L., Lv, Z.S. et al., 2017, 'Triazole derivatives and their anti-tubercular activity', European Journal of Medicinal Chemistry 138 501-513. https://doi.org/10.1016/j.ejmech.2017.06.051 